\title{
Two-dimensional fluid simulation of expanding plasma sheaths
}

\author{
MunPyo Hong and G. A. Emmert ${ }^{\text {a) }}$ \\ Department of Nuclear Engineering and Engineering Physics, University of Wisconsin, 1500 Johnson \\ Drive, Madison, Wisconsin 53706
}

(Received 24 October 1994; accepted for publication 30 August 1995)

\begin{abstract}
The transient sheath expansion around square and cross-shaped targets is simulated numerically with a two-dimcnsional fluid model. The angular distribution of the ions impinging on the target surface and the nonuniformity of the incident ion dose are calculated. The incident ion dose peaks near, but not at, the convex corner and has a minimum at the concave corner. The dip of the dose profile at the convex corner is shown to be caused by the product of a decreasing normal velocity profile and an increasing ion density profile along the target surface from the center to the corner. () 1995 American Institute of Physics.
\end{abstract}

\section{INTRODUCTION}

The issue of expanding sheaths has received renewed attention since the plasma-based ion implantation (PBII) process was invented by Conrad. ${ }^{1,2} \mathrm{PBII}$ is a new, non-line-ofsight technique for implanting ions into materials to modify their surface properties. ${ }^{2,3}$ In the PBII process, a target is immersed in a plasma and a high negative voltage pulse is applied. A sheath between the plasma and the target forms, and accelerates ions into the target. The ions are implanted in the target at energies up to the applied voltage. The implanted ions produce industrially relevant modification of the surface properties for a wide range of engineering materials and doping of semiconductors. Information concerning the dose, incident angle, and energy spectrum of the incident ions is needed to predict the modification of the surface and the effectiveness of the doping.

The expanding sheath has received considerable attention $^{4-6}$ for the study of ion acoustic waves. Because of Conrad's invention of PBII in 1986, the expanding sheath has more recently been discussed in the context of plasmabased ion implantation. ${ }^{7-11}$ Some successful results for onedimensional sheath evolution have been reported for numerical simulations ${ }^{9,10}$ and experiments. ${ }^{11}$

Recently, these efforts have been extended to multidimensional problems for application to real implantation processes which usually have complex-shaped targets. The effect of larget geometry on the structure of the sheath surrounding a multidimensional target has received some attention in practical PBII processing because geometrical effects induce spatial nonuniformity of the implanted ion dose and nonuniform implantation depth distribution caused by the nonperpendicular impact of ions. Donnelly and Watterson ${ }^{12}$ numerically solved the two-dimensional Poisson's equation for a stationary ion-matrix sheath near the corners of a convex and concave wedge-shaped target in a plasma. The steady state Child-Langmuir sheath structure near square and knife-shaped edges was simulated numerically by Watterson. ${ }^{13}$ This simulation assumed that all ions cross the interface between the sheath and the plasma at the same speed and number density, and perpendicular to the

\footnotetext{
a)Electronic mail: emmert@engr.wisc.edu
}

interface. They found that the implanted ion dose for the knife edge is more peaked than for a square edge. In addition, all ions strike the target with the same kinetic energy, but the angle of impact is nonperpendicular near the edge which reduces the implantation depth and increases sputtering.

More recently, Hong and Emmert ${ }^{14}$ have simulated the fully time-dependent, two-dimensional sheath expansion around a convex corner of a square target using a fluid model with a finite rise time voltage pulse. In that article, we reported that the incident dose is higher near the convex corner of the square target but has its maximum value at a slight distance from the corner, as seen in the steady state ChildLangmuir sheath simulation by Watterson. ${ }^{13}$ In addition, the angle at which the ions strike the surface is more oblique near the corner, and the area over which the oblique angle occurs broadens as the sheath grows. A similar calculation was performed for the same square geometry with a step pulse by Sheridan and Alport. ${ }^{15}$ They also found that the sheath focuses ions near to, but not on, the corner of the square target, resulting in an enhancement of the dose received there. They interpreted the dip of the ion dose profile at the corner of the square as caused by the finite inertia of ions, so that they are unable to follow the electric field; this causes a displacement of the ions away from the corner.

In this paper, we present numerical results of the twodimensional simulation for a cross-shaped target which has both convex and concave corners, using the same fluid model as in our previous article. ${ }^{14}$ The expanding sheath and geometry dependent parameters, such as the nonuniformity of the incident dose and the angular distribution of the ions impinging on the cross-shaped target are calculated. The nonuniformity of the incident dose and the nonperpendicular impact of the incident ions are of concern for the PBII process because they lead to nonuniform sputtering of the target, and nonuniform implantation depth and retained dose in the implanted layer. Additionally, we present results that explain the dip in the incident ion dose at the convex corner as being caused mainly by an increase in the angle of incidence and hence a decrease in the normal velocity of the ions near the corner. 


\section{FLUID MODELING AND FORMULATION}

For completeness the important features of the fluid model are briefly revicwed and the range of parameters investigated are described. We use two main assumptions: First, the ions are assumed to be collisionless and cold; they acquire directed motion only by the electric field. For the low pressures encountered in the PBII experiments of Shamim and co-workers, ${ }^{11}$ the collisionless ion approximation is reasonable. Next, the electrons are assumed to satisfy the Boltzmann relationship since electron inertiä effects can be neglected because of the small electron mass. This approximation allows the computation to jump over the electron time scale $\left(\sim 1 / \omega_{\text {pe }}\right)$ and solve the equations on the ion time scale $\left(\sim 1 / \omega_{\mathrm{p}}\right)$ which is the relevant time scale for the expansion of sheath and typically of the order of microseconds. Additionally, the effect of secondary electrons on the formation of the sheath structure is assumed to be negligible because secondary electrons are rapidly accelerated back into the bulk plasma where their density is negligible.

The compressible ion fluid equations in conservative form are

$$
\begin{aligned}
& \frac{\partial n_{i}}{\partial t}+\nabla \cdot\left(\dot{n}_{i} \mathbf{u}\right)=0 \\
& \frac{\partial}{\partial t}\left(n_{i} \mathbf{u}\right)+\nabla \cdot\left(n_{i} \mathbf{u u}\right)=-\frac{e n_{i}}{m_{i}} \nabla_{\phi} .
\end{aligned}
$$

The potential distribution is determined by Poisson's equation

$$
\nabla^{2} \phi=-\frac{e}{\epsilon_{0}}\left(n_{i}-n_{e}\right) .
$$

The electron density $n_{e}$ is given by the Boltzmann relation

$$
n_{e}=n_{0} \exp \left(e \phi / k T_{e}\right),
$$

where $n_{0}$ is the initial density in the plasma. Because the applied potential is large compared with the temperature, the electrons play no role in the sheath, but they are necessary to maintain quasineutrality in the plasma away from the sheath. Note that using the Boltzmann relation causes Poisson's equation to be nonlinear in the potential $\phi$; this complicates the solution algorithm.

It is convenient to nondimensionalize the ion fluid equations and Poisson's equation by introducing the following dimensionless variables:

$$
\begin{aligned}
& \mathbf{R}^{*}=\mathbf{R} / L \text { or } \nabla^{*}=L \nabla, \\
& \mathbf{u}^{*}=\mathbf{u} / c_{s}, \\
& t^{*}=t /\left(L / c_{s}\right), \\
& n_{i}^{*}=n_{i} / n_{0} \\
& \psi=e \phi / k T_{e},
\end{aligned}
$$

where $c_{s}$ is the ion acoustic speed defined as $\left(k T_{e} / m_{i}\right)^{1 / 2}$, and $L$ refers to the size of the plasma being simulated. Dropping asterisks, the system of equations becomes

$$
\begin{aligned}
& \frac{\partial n_{i}}{\partial t}+\nabla \cdot\left(n_{i} \mathbf{u}\right)=0, \\
& \frac{\partial}{\partial t}\left(n_{i} \mathbf{u}\right)+\nabla \cdot\left(n_{i} \mathbf{u u}\right)+n_{i} \nabla \psi=0, \\
& \nabla^{2} \psi=-\left(L / \lambda_{D}\right)^{2}\left(n_{i}-e^{\psi}\right),
\end{aligned}
$$

where $\lambda_{D}=\left(\epsilon_{0} k T_{e} / n_{0} e^{2}\right)^{1 / 2}$ is the Debye length. Equation (7) is solved by finite differencing and the full multigrid (FMG) method. To solve for the ion density and velocity from Eqs. (5) and (6), we use a fully implicit finite-difference scheme with an adequate mesh size to avoid numerical instability or numerical oscillations. ${ }^{16}$ These oscillations are the result of inadequate mesh refinement in regions of large gradients near the target surface, i.e., violation of the Courant-Friedrichs-Lewy (CFL) stability condition. ${ }^{16} \mathrm{We}$ choose the Beam and Warming algorithm, ${ }^{16-18}$ which is second-order time-accurate, noniterative in a spatially factored form, and unconditionally stable. The entire scheme (Poisson solver and fluid solver) can be unstable even though each solver is unconditionally stable; the entire stability of our numerical strategy composed explicitly of the Poisson solver and the fluid solver is dependent on the mesh size, CFL number, and the coefficient of the explicit artificial damping term. Our numerical procedure is very stable for adequate numerical parameters. A fourth-order explicit artificial dissipation term was added in the fluid equations to suppress high frequency oscillations without reducing accuracy. ${ }^{16}$ The finite difference scheme for the fluid equation conserves particles to an accuracy of the order of $10^{-8}-10^{-9}$ per cell.

The Poisson solver and the fluid solver operate alternately to calculate the transient sheath expansion for the simulation of a PBII pulse. The resulting code runs satisfactorily on a UNIX-based workstation.

\section{SIMULATION FOR CROSS-SHAPED TARGET}

A cross-shaped target (shown in Fig. 1) having both convex and concave corners was considered. The total width of this target is $16 \mathrm{~cm}$ and the depth of the concave side is $4 \mathrm{~cm}$. The outer boundary of the plasma has a square shape with a half-width $(L)$ of $25.75 \mathrm{~cm}$. Because of symmetry, we calculated the sheath expansion in only one quadrant shown as a cross-hatched area. The applied potential is zero initially and rises (in the negative sense) at a rate of $6.0 \mathrm{kV} / \mu$ s until reaching the plateau value of $-30 \mathrm{kV}$. The plasma is argon having a density of $3.0 \times 10^{9} \mathrm{~cm}^{-3}$; and the electron temperature is $2.0 \mathrm{eV}$. For this calculation, the ion acoustic speed $c_{s}$ is $0.22 \mathrm{~cm} / \mu$ s and the Debye length $\lambda_{D}$ is $0.0192 \mathrm{~cm}$. The plasma half-width is 1341 Debye lengths. In addition, the maximum Mach number $u / c_{s}$ is 173 and the pulse time of 15 $\mu$ s corresponds to 0.13 units of $L / c_{s}$ or 27 ion plasma periods. A square mesh system was used having a mesh size of $0.0625 \mathrm{~cm} \quad\left(=3.26 \lambda_{D}\right.$-the effect of smaller mesh sizes relative to the Debye length is discussed in Sec. IV) and time step is automatically optimized ( $d t \equiv \mathrm{CFL} d x$ ) $\left.\left(2 e|V(t)| / m_{i}\right)^{1 / 2}\right)$, where $V(t)$ is the applied potential at a given time, and the CFL number is taken to be 0.8 . 


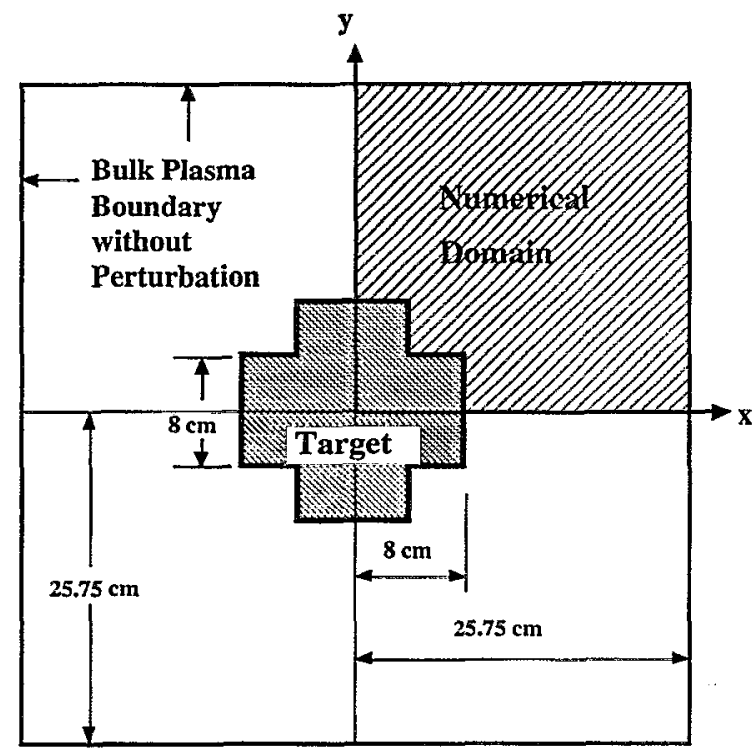

FIG. 1. Geometry of the system with a cross-shaped target.

Figures 2(a)-2(c) show that the behavior of the sheath evolution around the cross-shaped target is qualitatively the same as that around a square target. ${ }^{14}$ The sheath edge initially conforms to the target and becomes almost cylindrical in the long time limit. In these simulations the shape of the target hardly affects the shape of the sheath edge in the later time stage. This is because the sheath thickness becomes larger than the target dimension. This characteristic of the plasma sheath has also been observed in experiments by Lim et al. ${ }^{19}$ Note that the jaggedness in the ion density profile at the sheath edge [as shown in Fig. 2(c)] is a numerical artifact caused by a shock-wave-nature of the density profile. ${ }^{20}$

The potential contours are plotted in Figs. 3(a)-3(c) at several time steps to make a comparison in the sheathevolution rate near the convex corner and near the concave corner. The expansion of the sheath is fastest at the concave corner and slowest at the convex corner, as we expected.

The temporal evolution of the angle, relative to the normal, at which the incident ions strike the surface of the crossshaped target is shown in Fig. 4. The negative value of the distance from the tip point of the convex corner refers to the outside face of the convex corner, and the positive value refers to the inside face near the concave corner. As expected, ions striking the inside area of the concave corner have a larger angle of incidence which becomes more pronounced as the pulse progresses. Eventually, most of the ions striking the inside hit the surface with angles of about $45^{\circ}$. On the other hand, the trend of incident angles on the outside face is similar to the previous square target case,${ }^{14}$ except for the variation of the local maximum value at the convex corner itself with relation to time. Initially, the angle of incidence has the same value of $45^{\circ}$ at both corners. As time increases, the incident ions become less oblique on the outside face very near the convex corner and more oblique further away from the corner. On the contrary, in the inside face near the convex corner, the incident angle becomes larger as the sheath evolves to the cylindrical shape. Note that a dis-

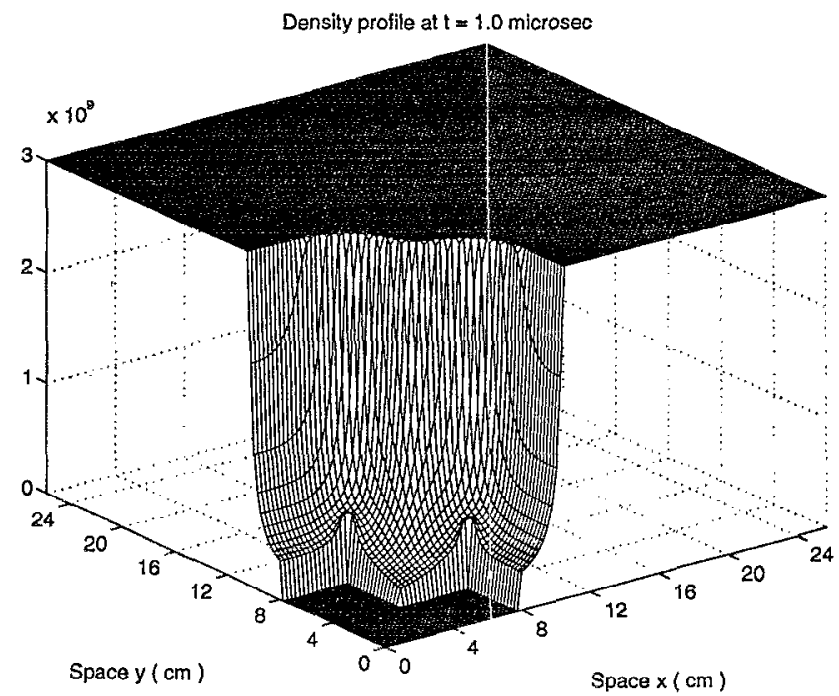

(a)

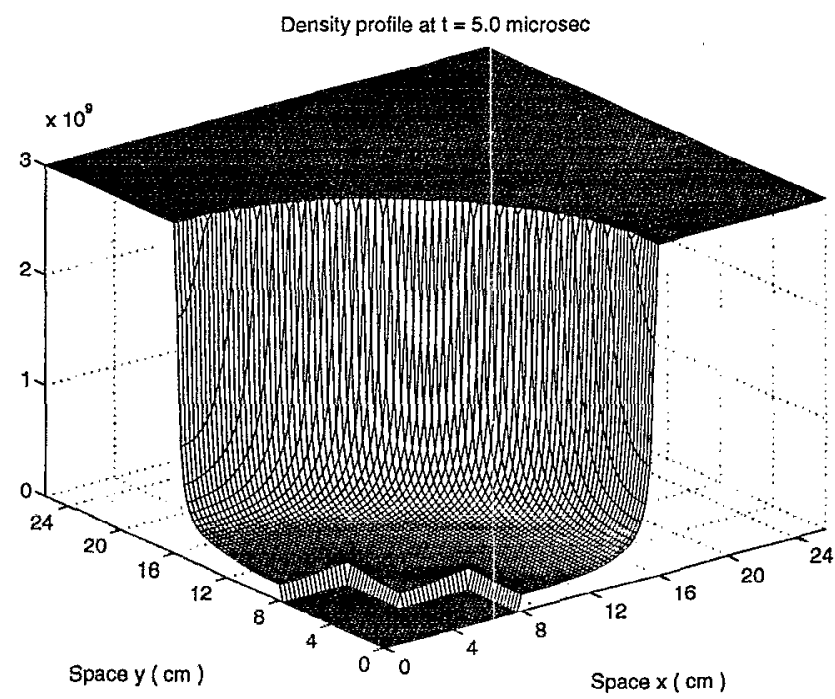

(b)

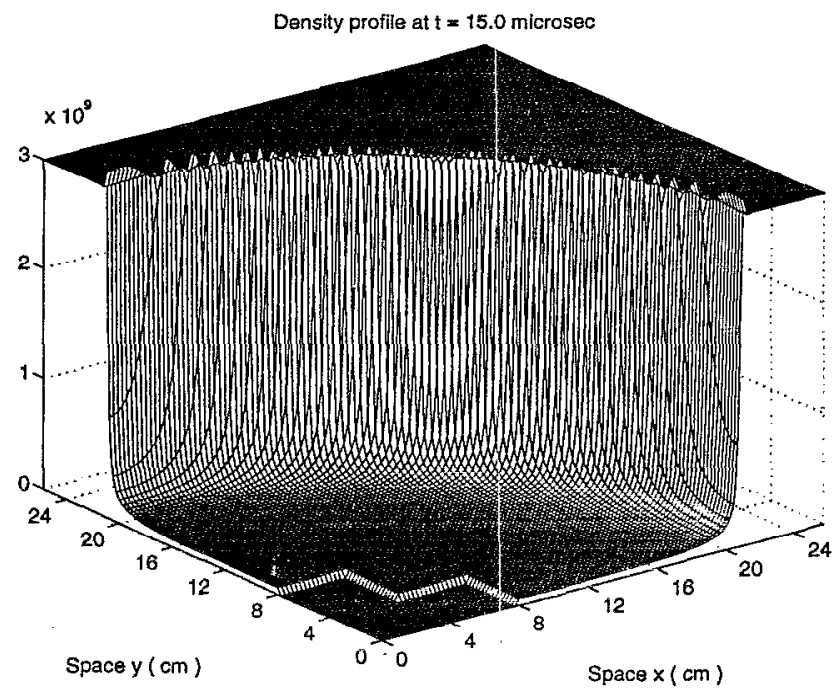

(c)

FIG. 2. Evolution of the ion density around the cross-shaped target. 

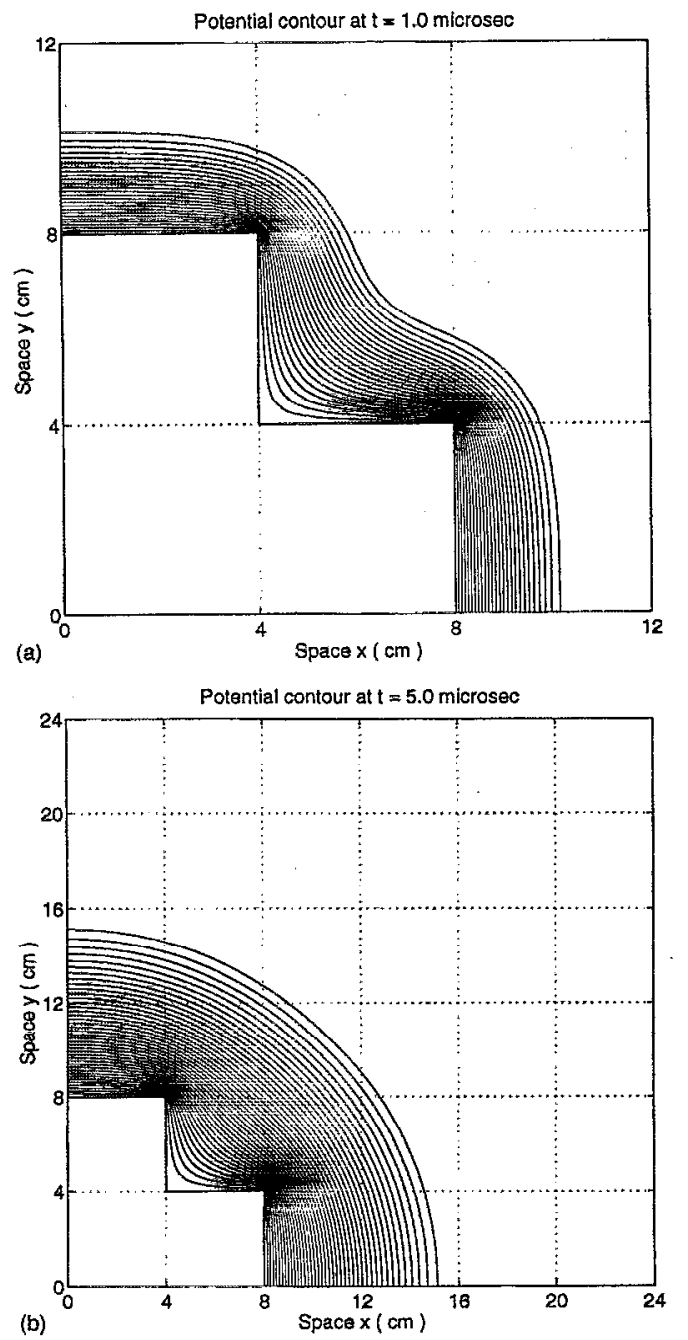

(b)

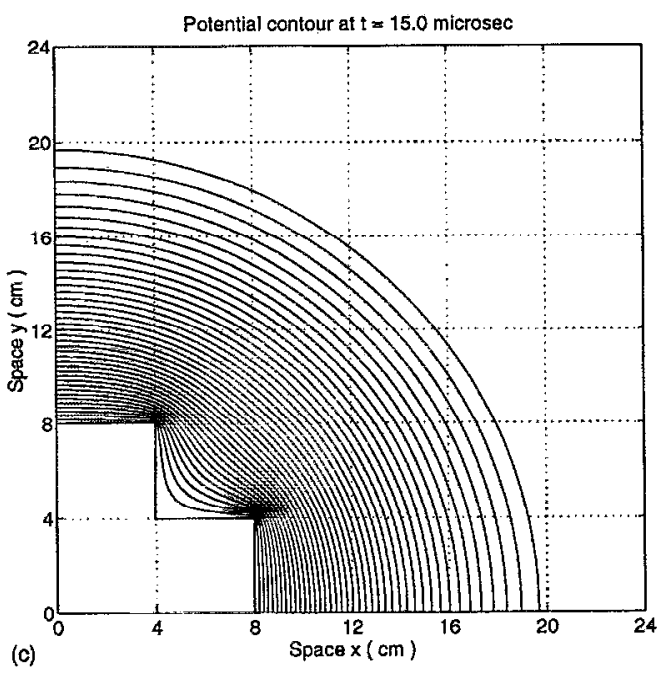

FIG. 3. The sheath potential contours around the cross-shaped target at various times.

continuity appears at the tip of the convex corner; this is caused by the definition of the incident angle and the sudden change in the orientation of the surface. The velocity is continuous at the corner.

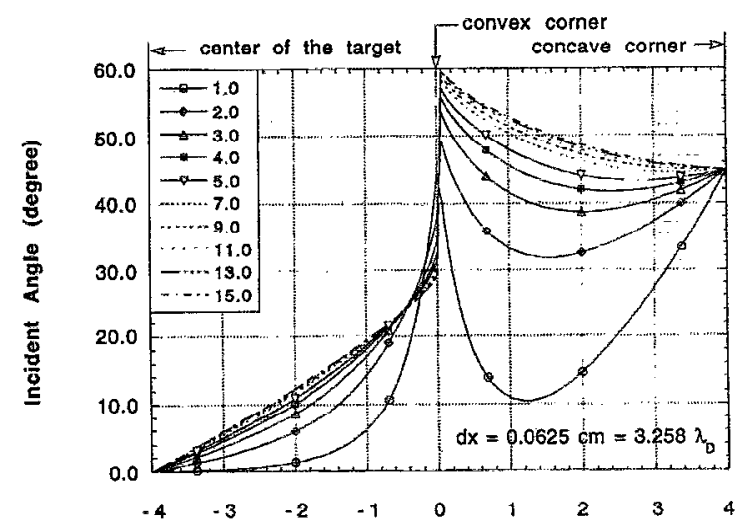

Distance from the convex cornet of the cross-shaped target $(\mathrm{cm})$

FIG. 4. The angle at which the incident ions strike the cross-shaped target surface at various times during the pulse. The negative value of the distance denotes the outside face, and the positive value denotes the face between the convex and concave corners. Not all data points are shown.

The temporal evolution of the incident ion dose profile is presented in Fig. 5. The incident ion dose $D(x, y)$ is given by a time integral over the pulse of the ion flux at the target surface

$$
D(x, y) \equiv \int_{t_{0}}^{t_{p}} \Gamma_{i}(t, x, y) d t=\int_{t_{0}}^{t_{p}} n_{i}(t, x, y) u_{\perp}(t, x, y) d t,
$$

where $t_{0}$ is the start time of the pulse, $t_{p}$ is the pulse duration and $u_{\perp}$ is the ion speed normal to the target surface. The incident dose is higher near the convex corner and lower near the concave corner. The nonuniformity of the dose in the cross-shaped target is more severe than that in the simple square target. The dip of the dose at the convex corner seen in previous articles ${ }^{13-15}$ is apparent. This dip effect at the tip point is discussed to a greater extent in the next section.

\section{DISCUSSION OF THE DIP EFFECT}

The numerical results presented in Fig. 5 stimulate the following questions: Why doesn't the peak of the incident

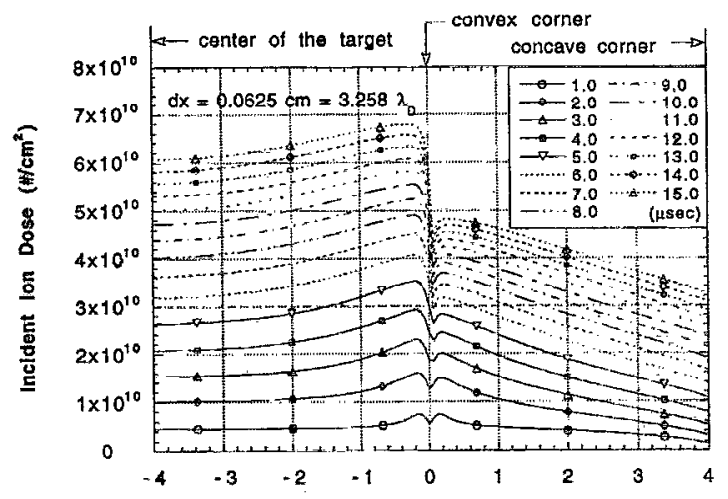

Distance from the convex corner of the cross-shaped target $(\mathrm{cm})$

FIG. 5. The incident ion dose profile on the cross-shaped target at various times during the pulse. 


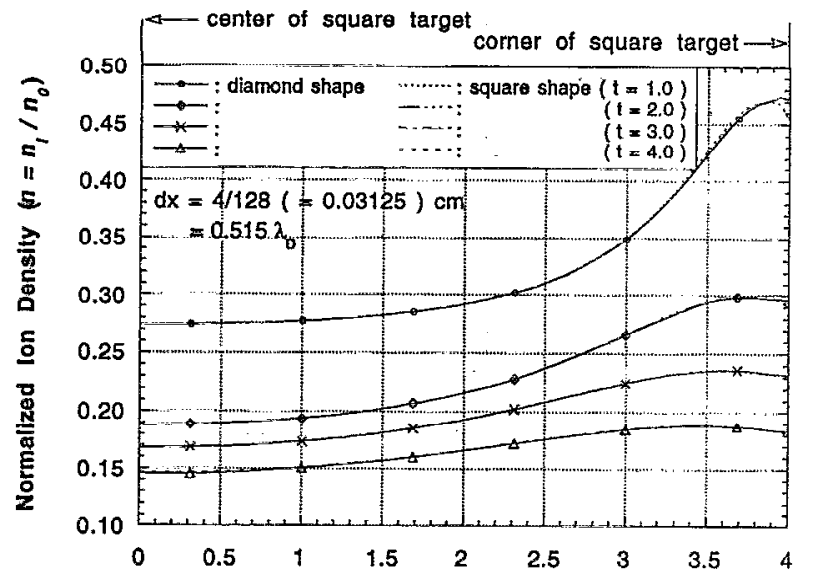

Distance from the center of the square target $(\mathrm{cm})$

FIG. 6. Comparison of the ion density profiles for the square target (dotted line) and the diamond-shaped target (solid line) at various times. The positions $x=0.0$ and $4.0 \mathrm{~cm}$ refer to the center and the corner of the square target, respectively.

dose appear at the convex corner itself? What is the reason for the dip at the corner? In this section these questions are considered.

To determine the reason for this effect, additional calculations for the square target problem described in the our previous article have been carried out. ${ }^{14} \mathrm{~A}$ finer mesh was used than in earlier calculations to better resolve the dip. The numerical procedure and boundary conditions are the same as for previous calculations for the cross-shaped target, but the plasma parameters and simulation conditions are somewhat different. The width of the square target is $8 \mathrm{~cm}$. The argon plasma has a density of $3.0 \times 10^{8} \mathrm{~cm}^{-3}$ and the electron temperature is $2.0 \mathrm{eV}$. For this simulation, the ion acoustic speed $c_{s}$ is $0.22 \mathrm{~cm} / \mu \mathrm{s}$, and the Debye length $\lambda_{D}$ is 0.0607 $\mathrm{cm}$. The square target is 132 Debye lengths wide. The applied pulse rise rate (in the negative sense) is $3.333 \mathrm{kV} / \mu \mathrm{s}$ until the voltage reaches the plateau value of $-10 \mathrm{kV}$. A pulse time of $4 \mu \mathrm{s}$ corresponds to 2.3 ion plasma periods. A square mesh system with a spacing of $0.03125 \mathrm{~cm}(0.515$ $\left.\lambda_{D}\right)$ is used.

Since the incident ion dose $D$ is defined as total time integral over the pulse of the incident flux at the target surface, the role of the ion density and the normal component of the ion velocity should be considered separately. As shown in Fig. 6, the ion density at the square target is also enhanced near the corner. This is caused by the electric field focusing toward the convex corner. The density profile for a square target (dotted line) in Fig. 6 shows a small drop near the corner.

In Fig. 7, the incident ion dose profile for different mesh sizes of the square target with $8 \mathrm{~cm}$ width at $t=2.0 \mu \mathrm{s}$ is compared. Simulations with a mesh sizc of twice the Debye length $\lambda_{D}$ give reasonable results, except for poor resolution near the corner. Calculations very near the corner are subject to numerical errors because of the nature of the corner and the sharp change in the shape of the electric field lines.

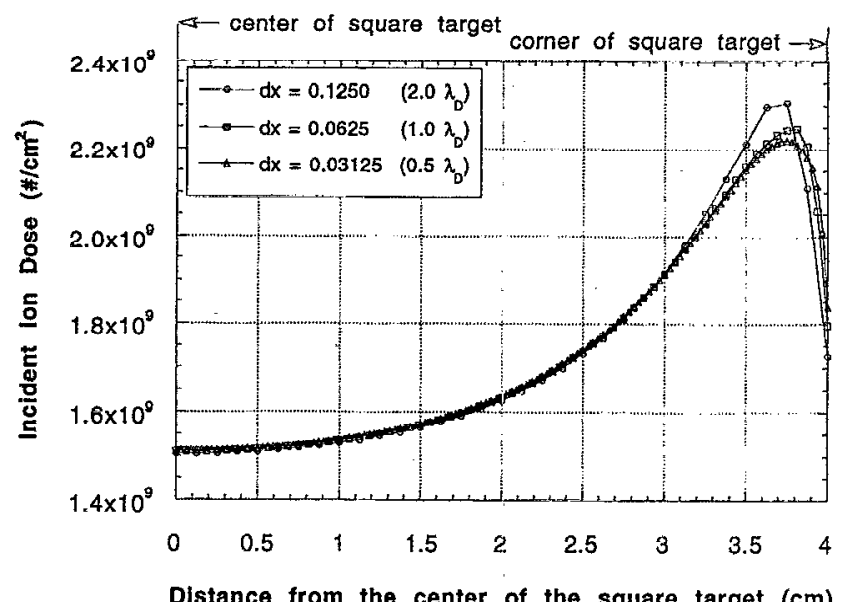

FIG. 7. The incident ion dose profile for different mesh sizes on the square target at $t=2.0 \mu \mathrm{s}$. The position $x=4 \mathrm{~cm}$ indicales the corner of the target.

In addition to resolution, numerical diffusion (false diffusion) near the corner concerned us. False diffusion is a purely numerical phenomenon in multidimensional problems and is caused by the numerical scheme for a highly convective fluid system; ${ }^{21}$ this numerical diffusion occurs when the flow is oblique to the grid line and when there is a nonzero gradient of the dependent variable in the direction orthogonal to the flow, as shown in Fig. 8. An approximate expression for the false diffusion coefficient for a two-dimensional situation has been given by de Vahl, Davis, and Mallinson ${ }^{21}$

$$
\mu_{\mathrm{false}}=\frac{\rho u \Delta x \Delta y \sin 2 \theta}{4\left(\Delta y \sin ^{3} \theta+\Delta x \cos ^{3} \theta\right)},
$$

where $u$ is the fluid velocity and $\theta$ is the angle between the velocity vector and the axis normal to the target. Equation (9) shows that false diffusion is not present when the resultant flow is along one of the sets of grid lines $\left(\mu_{\mathrm{false}^{-}}{ }^{-0}\right)$; on the other hand, false diffusion is more serious when the flow direction makes an angle of $45^{\circ}$ with the grid lines.

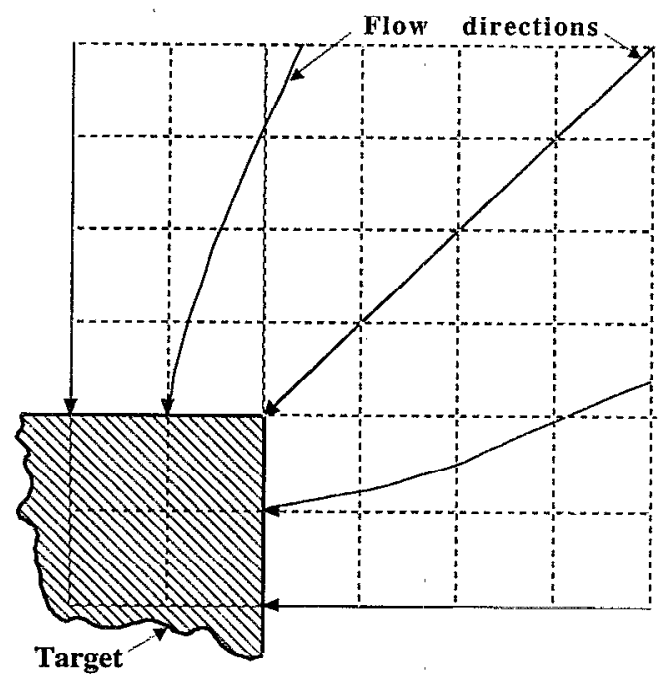

FIG. 8. Flow directions for the square target. 


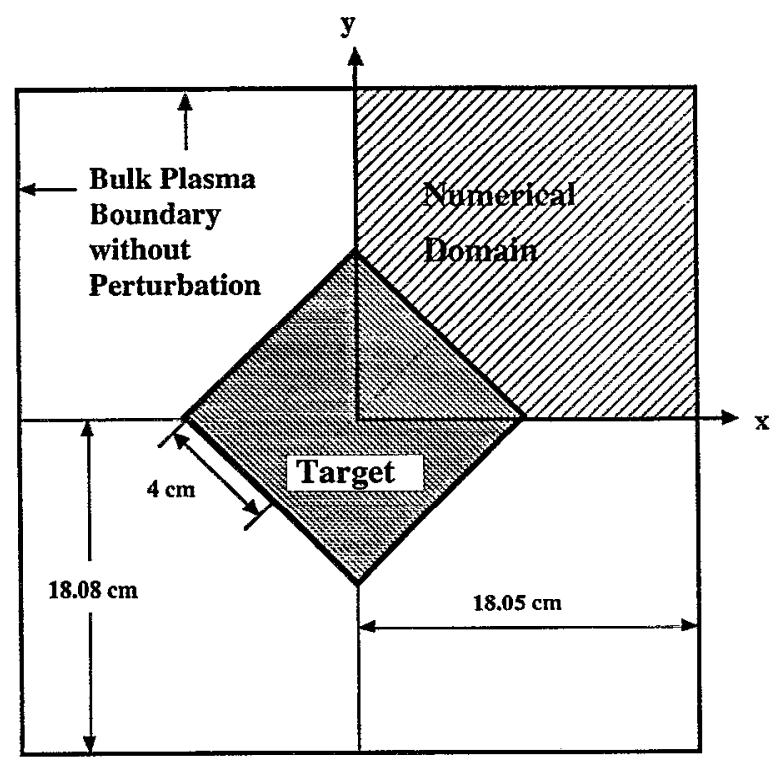

FIG. 9. Geometry of the system with a diamond-shaped target; the square target is rotated by $45^{\circ}$ and tip points of the convex corners are aligned on the $x$ and $y$ axes.

Unfortunately, because of the changing flow direction, our fluid solver can not avoid the condition for false diffusion; near the corner, the flow will be at $45^{\circ}$ to the mesh. To make matters worse, the ion density is enhanced near the corner of the target due to the focusing of ions. Consequently, along the flows toward the corner, the ion density will be underestimated since the ion flow suffers from numerical diffusion which transports ions away from the corner.

To reduce false diffusion near the corner, the square target configuration was rotated by $45^{\circ}$ (called a "diamondshaped target") and the flow direction at the corner was aligned with a grid line as shown in Fig. 9. The $45^{\circ}$ rotation not only reduces false diffusion but also gives a more reasonable estimate of the electric field at the corner.

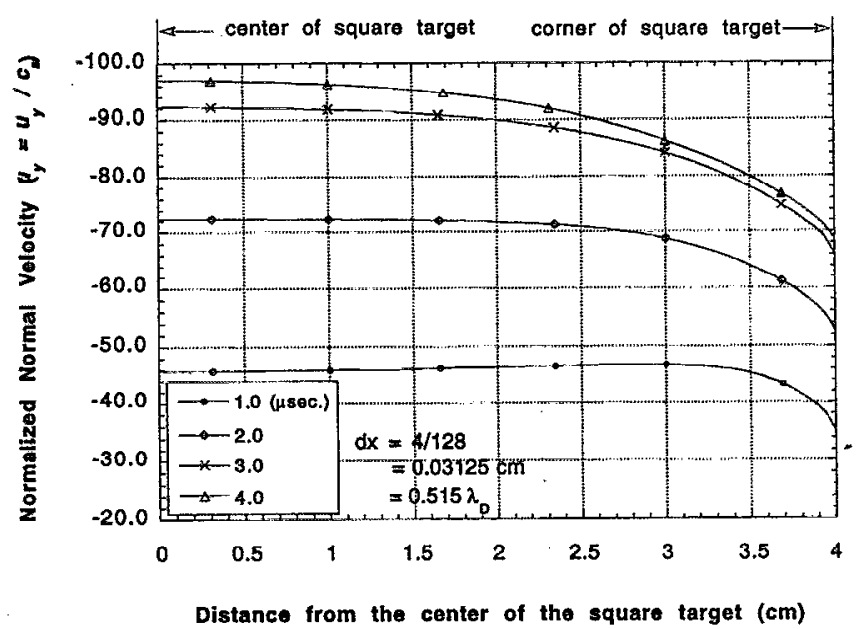

FIG. 10. The normalized normal velocity of the incident ion at the square target surface at various times during the pulse.

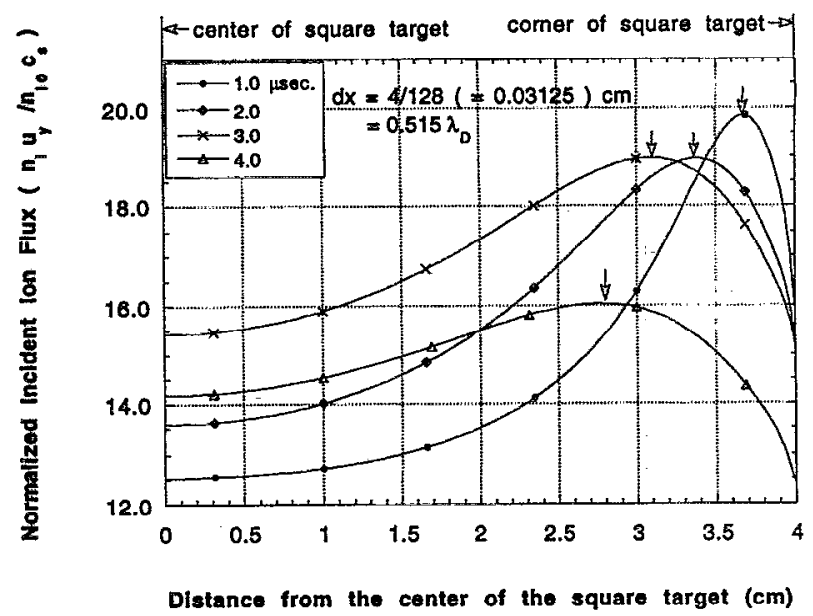

FIG. 11. The normalized incident ion flux profile on the square target at various times during the pulse.

Figure 6 also shows the comparison of the evolution of the ion density profile for the square target and the diamondshaped target (rotated square target) with the same simulation conditions. The dotted line and the solid line refer to the ion density profile at the target surface for the square target and the diamond-shaped target, respectively. In the ion density profile for the diamond-shaped target, the dip of the ion density is mitigated near the corner of the target for early times in the pulse.

Similarly, the normal component of the ion velocity at the target surface decreases monotonically as the corner is approached. This is shown in Fig. 10. Larger angle of incidence of the ions hitting near the corner causes this monotonic trend; at the corner, the angle of incidence approaches $45^{\circ}$. The velocity profiles (total speed and normal velocity) are identical for the square target and diamond-shaped target cases.

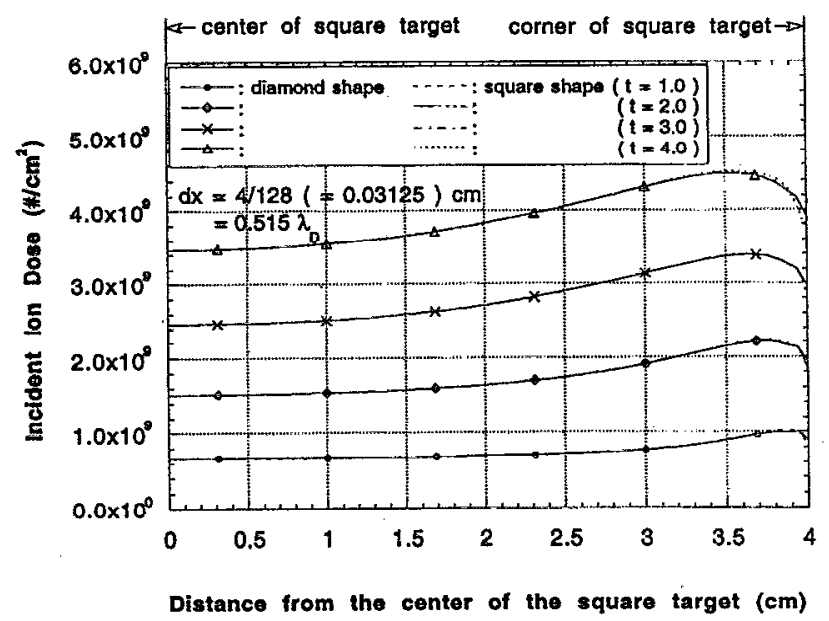

FIG. 12. Comparison of the incident ion dose profiles for the square target (dotted line) and the diamond-shaped target (solid line) at various times. The positions $x=0.0$ and $4.0 \mathrm{~cm}$ refer to the center and the corner of the square target, respectively. 
Figure 11 shows that the ion flux (product of the ion density and the normal speed of ions) on the surface of the square target is not monotonic, but achieves a local maximum, the location of which moves away from the corner as the pulse progresses.

The incident ion dose, i.e. time-integrated incident ion tlux, is presented in Fig. 12. The dip near the corner appears in both the square and diamond cases with essentially the same results, even very near the corner. The variation of ion density near the corner is not a large factor in the dose profile near the corner. The most important factor for peaking of the incident ion dose near the corner is the decrease in the normal component of the incident ion velocity because of the angle at which the ions strike the surface.

\section{SUMMARY}

We have simulated numerically the evolution of the transient sheath around a cross-shaped target, which has both convex and concave corners, using a two-dimensional fluid model. The angular distribution of the ions impinging on the target surface and the incident ion dose profile are calculated. A dip of the dose profile still appears at the convex corner of the cross-shaped target. We investigate the reason for the dip effect, and show that it is a local maximum caused by the product of an increasing ion density profile and a monotonically decreasing normal velocity profile along the target surface from the center to the corner.

\section{ACKNOWLEDGMENTS}

This work was supported by the DOE CRADA Agreement No. 9-XX3-8362-E-1 and the U.S. Army Research Office Contract No. DAALO3-90-G-0124.

'J. R. Conrad, U.S. Patent No. 4,764,394 (August 1988).

${ }^{2}$ J. R. Conrad, J. L. Radke, R. A. Dodd, F. J. Worzala, and N. C. Tran, J. Appl. Phys. 62, 4591 (1987).

${ }^{3}$ J. R. Conrad, R. A. Dodd, S. Han, M. Madapura, J. Scheuer, K. Sridharan, and F. J. Worzala, J. Vac. Sci. Technol. A 8, 3146 (1990).

${ }^{4}$ M. Widner, I. Alexeff, W. D. Jones, and K. E. Lonngren, Phys. Fluids 13, $2532(1970)$.

${ }^{5}$ J. K. Chester, J. Sci. Technol. 37, 2 (1970).

${ }^{6}$ G. Andrews and R. H. Varey, Phys. Fluids 14, 339 (1971).

${ }^{7}$ J. R. Conrad, J. Appl. Phys. 62, 777 (1987).

${ }^{8}$ M. A. Lieberman, J. Appl. Phys. 66, 2927 (1989).

${ }^{9}$ J. T. Schener, M. Shamim, and J. R. Conrad, J. Appl. Phys. 67, 1241 (1990).

${ }^{10}$ G. A. Emmert and M. A. Henry, J. Appl. Phys. 71, 113 (1992).

${ }^{11}$ M. Shamim, J. T. Scheuer, and J. R. Conrad, J. Appl. Phys. 69, 2904 (1991).

${ }^{12}$ I. J. Donnelly and P. A. Watterson, J. Phys. D 22, 90 (1989).

${ }^{13}$ P. A. Watterson, J. Phys. D 22, 1300 (1989).

${ }^{14}$ M. P. Hong and G. A. Emmert, J. Vac. Sci. Technol. B 12, 889 (1994).

${ }^{15}$ T. E. Sheridan and M. J. Alport, Appl. Phys. Lett. 64, 1783 (1994).

${ }^{16}$ D. A. Anderson, J. C. Tannehill, and R. H. Pletcher, Computational Fluid Mechanics and Heat Transfer (McGraw-Hill, New York, 1984), Chap. 9.

${ }^{17}$ R. M. Beam and R. F. Warming, J. Comp. Phys. 22, 87 (1976).

${ }^{18}$ R. M. Beam and R. F. Warming, AIAA J. 16, 393 (1978).

${ }^{19}$ W. B. Lim, J. Cooney, M. S. Ghaemi, M. Gavin, D. R. Andesen, and K. E. Lonngren, J. Phys. D 24, 1081 (1991).

${ }^{20}$ R. J. LeVeque, Numerical Methods for Conservation Laws, Lectures in Mathematics ETH Zurich (Birkhauser, Basel, 1994), pp. 114-135.

${ }^{21}$ S. V. Patankar, Numerical Heat Transfer and Fluid Flow (McGraw-Hill, New York, 1980), pp. 105-109. 